\title{
Understanding CpG methylation in the context of osteoarthritis
}

\author{
"...interactions between the genome and the epigenome, and resultant \\ allele-specific DNA methylation, could underpin much of the etiology, and possibly \\ heritability, of osteoarthritis."
}

\section{KEYWORDS: cartilage " epigenetic inheritance " genetic susceptibility $\approx$ methylation - osteoarthritis}

Worldwide, osteoarthritis (OA) affects approximately $40 \%$ of people older than 70 years and is therefore the most common form of arthritis. OA is a complex degenerative disease of the joint but is epitomized by focal degradation of articular cartilage [1]. Cartilage itself is a highly specialized tissue composed of an extracellular matrix (ECM) rich in type-II collagen and the proteoglycan aggrecan. The tissue is sparsely populated by the sole resident cell, the chondrocyte, which maintains tissue integrity by balancing the synthesis and degradation of ECM components [2]. In $\mathrm{OA}$, this balance is perturbed in favor of ECM degradation, mediated by proteolytic enzymes including MMP13 and ADAMTS5, which probably degrade the type-II collagen and aggrecan, respectively [3]. Inhibition of metalloproteinase activity should protect against cartilage destruction, but has failed in clinical trials in part owing to nonselectivity and resulting adverse side effects [4]. Amongst other things, this has led to the suggestion that targeting the regulation of expression of specific proteases, or regulating factors, could be an important therapeutic option to slow or halt $\mathrm{OA}$ progression. There is an urgent need for such disease-modifying, noninvasive treatments for OA since the only current therapy is joint replacement surgery.

Epigenetic regulation involves three basic mechanisms: DNA methylation, histone modifications and ncRNAs. DNA methylation represents the most stable epigenetic modification and involves the addition of a methyl group to the cytosine at $\mathrm{CpG}$ dinucleotides, converting cytosine to 5-methylcytosine. This functions to disrupt the binding of transcription factors or enhance the binding of repressors, leading to an altered transcriptional state of the cell [5]. In terms of OA research, a number of studies have investigated the role that epigenetics, especially DNA methylation, could play in OA, particularly in terms of regulating the balance between matrix synthesis and degradation in cartilage. These studies have focused on correlating DNA methylation status with gene expression at a few candidate genes (loci), including those coding for aggrecan, collagens, MMPs, LEPTIN, SOX9 and RUNX2 [6]. Broadly, these works have shown a correlation between promoter methylation and the expression of the associated gene. Recently, we took this work one step further and identified a specific differentially methylated $\mathrm{CpG}$ locus within the MMP13 promoter whose methylation status regulates the expression of the gene by controlling the binding of the transcriptional regulator CREB [7]. These and ongoing 'candidate' gene studies will continue to identify differentially methylated regions that may be important in onset of the disease.

"...targeting the regulation of expression of specific proteases, or regulating factors, could be an important therapeutic option to slow or halt osteoarthritis progression."

OA has a multifactorial etiology and is a consequence of the interaction of local and systemic risk factors, especially genetics [8]. Heritability studies suggest approximately $50 \%$ of the risk of developing OA can be explained by genetics; however, despite extensive efforts only a few loci have been associated with $\mathrm{OA}$ at genome-wide significance levels, and even these signals have a limited effect size [9]. One likely reason for the paucity of identified genetic susceptibility loci for OA could be low penetrance polymorphisms in the population that remain to be identified and studies are ongoing to identify these. However, it is proposed that, in part, this missing genetic heritability may be accounted for by inheritance of epigenetic modifications. However, in mammals evidence for

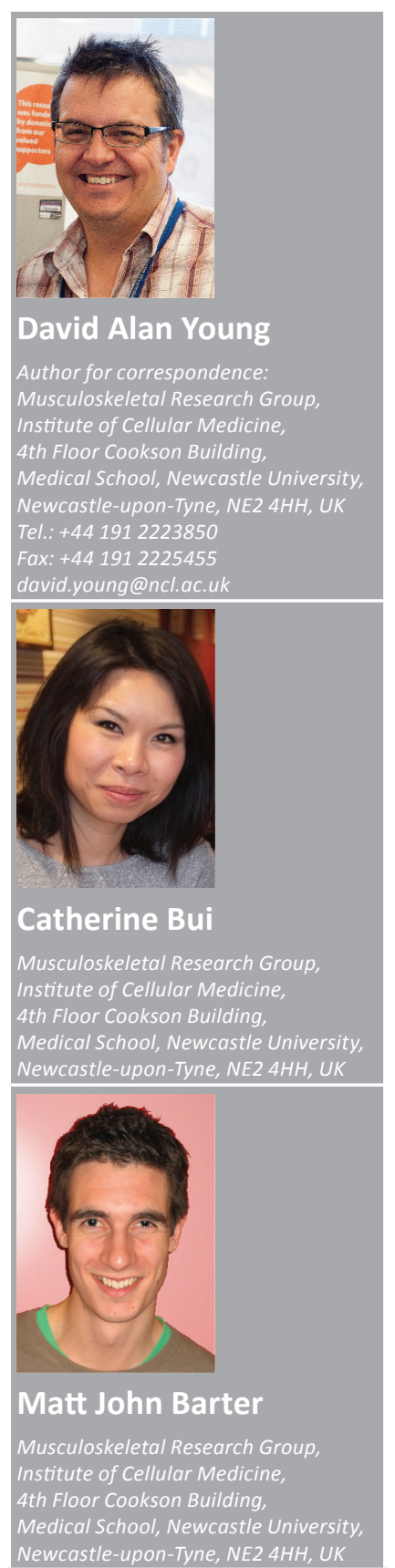

Future $\mathrm{fSS}$ 
such epigenetic transgenerational inheritance is limited, although more studies are alluding to the possibility [10]. The reason such transgenerational inheritance is unlikely to be common is because there is an almost complete erasure of DNA methylation during the formation of primordial germ cells (PGCs; which give rise to the gametes) from a subset of posterior proximal epiblast cells early in embryonic development. This resetting of the genomic imprinting pattern is essential in the reprogramming of the developmental potential of PGCs to a totipotent state [11]. In fact during this reprogramming, only approximately $7 \%$ of $\mathrm{CpGs}$ remain methylated with these $\mathrm{CpGs}$ clustering at regions of recently acquired retrotransposons and potentially functioning to ensure transposable element suppression and hence maintenance of genomic integrity in the germline. The complete mechanism of demethylation in PGCs is elusive but probably partly involves the conversion of 5-methylcytosine to the recently identified 5-hydroxymethylcytosine and back to cytosine via the base excision repair pathway [11].

\section{"...the genome can influence the epigenome and potentially disease penetrance."}

One would assume then that epigenetics, at least DNA methylation, cannot explain the missing OA heritability. However, studies examining methylation patterns in monozygotic and dizygotic twins confirm the long-standing suggestion that the genome influences the epigenome. DNA methylation patterns between monozygotic twins correlate more than those between dizygotic twins, plus methylation patterns are more comparable between cotwins than between unrelated pairs of individuals [12]. These data indicate a degree of DNA methylation heritability, even if this is due to underlying genome heritability. Of course, since the twin concordance rate for a complex disease never reaches $100 \%$ this too suggests a potential role for epigenetics via, in part, environmental factors. Epidemiological studies indicate that such environmental events in utero can impact on disease outcomes. These findings do not necessarily demonstrate true transgenerational inheritance but reveal how the genome can influence the epigenome and potentially disease penetrance. Furthermore, new techniques in nucleic acid sequencing have begun to reveal the level of allele-specific methylation (ASM) within the genome [13]. Across the mammalian genome the level of DNA methylation was presumed to be complementary on both alleles, except for several well known examples, such as the X chromosome, but recent studies now indicate that ASM may be relatively widespread. ASM is most frequently associated with the local genotype, that is, cis ASM, which would be inherited in a Mendelian manner and thus provide an epigenetic mechanism where a noncoding sequence variation can have a phenotypic effect. More interesting perhaps is the phenomena of non-cis ASM, predicted to be due to transacting factors, stochastic events or parental-origin-dependent imprinting. For example, in Type 2 diabetes a SNP (rs2334499) is protective but only when maternally inherited. The SNP correlates with the differential methylation (and binding of the factor CTCF) at a nearby locus regardless of whether the allele was inherited from the father or the mother [14]. Although, perhaps only encompassing $10 \%$ of all ASM, non-cis ASM can cause problems for genomewide association studies (GWAS) since it renders loci effectively hemizygous and dilutes or breaks allelic association. Thus, such non-cis ASM has been proposed to account for at least a proportion of the missing heritability in common complex human diseases [13], perhaps including OA.

\section{"Whether epigenetic mechanisms can explain the missing heritability of common diseases, such as osteoarthritis, remains to be determined [but] DNA methylation will undoubtedly play a pivotal role in the development and maintenance of a stable-differentiated chondrocyte phenotype."}

For now, we would suggest that OA research concentrates on the emerging area of understanding how the complete repertoire of epigenetic effects, including chromatin modifications and ncRNAs, can impact on the penetrance of genetic susceptibility, with initial work focusing on the identified OA susceptibility alleles [9]. Such experiments involve the integration of genetic and epigenetic data, which could help explain and add weight to current GWAS signals [15]. To illustrate this, we recently showed that one of the genes genetically associated with OA, GDF5, was also epigenetically regulated [16]. A common SNP rs143383 within the 5'-UTR of GDF5 is associated with OA and is functionally involved in the expression of the gene. This SNP is a C to T transition and, when a C, forms part of a $\mathrm{CpG}$ dinucleotide and is therefore potentially subject to methylation. Indeed, we observed that the differential expression of GDF5 with the T versus $\mathrm{C}$ allele was modulated by DNA methylation changes, demonstrating 
the complex relationship and potential synergy between genetics and epigenetics at an OA susceptibility locus. As with other complex diseases, we would hypothesise that interactions between the genome and the epigenome, and resultant allele-specific DNA methylation, could underpin much of the etiology, and possibly heritability, of OA. With the release of the largescale ENCODE data sets, it is now possible to identify whether disease-associated noncoding SNPs identified from GWAS reside in regulatory regions (i.e., DNase hypersensitive loci/enhancers) of the genome; areas themselves that are subject to regulation by DNA methylation [17].

Whether epigenetic mechanisms can explain the missing heritability of common diseases, such as $\mathrm{OA}$, remains to be determined. Although under investigation, DNA methylation will undoubtedly play a pivotal role in the development and maintenance of a stable-differentiated chondrocyte phenotype. Moreover, it seems clear that epigenetic changes that accumulate over years owing to, for example diet or aging mechanisms, may well be important in modulating the deleterious or advantageous effects of a gene in OA. Owing to the reversibility of these DNA methylation marks, the potential for sequence-specific targeted therapeutics does exist.

Finally, cartilage has been the joint tissue at the center of most OA research but some believe

\section{References}

1 Goldring SR, Goldring MB. Clinical aspects, pathology and pathophysiology of osteoarthritis. J. Musculoskelet. Neuronal Interact. 6(4), 376-378 (2006).

2 Goldring MB. Molecular regulation of the chondrocyte phenotype. J. Musculoskelet. Neuronal Interact. 2(6), 517-520 (2002).

3 van den Berg WB. Osteoarthritis year 2010 in review: pathomechanisms. Osteoarthritis Cartilage 19(4), 338-341 (2011).

4 Murphy G, Nagase H. Reappraising metalloproteinases in rheumatoid arthritis and osteoarthritis: destruction or repair? Nat. Clin. Pract. Rheumatol. 4(3), 128-135 (2008).

5 Klose RJ, Bird AP. Genomic DNA methylation: the mark and its mediators. Trends Biochem. Sci. 31(2), 89-97 (2006).

6 Barter MJ, Bui C, Young DA. Epigenetic mechanisms in cartilage and osteoarthritis: DNA methylation, histone modifications and microRNAs. Osteoarthritis Cartilage 20(5), 339-349 (2012).

7 Bui C, Barter MJ, Scott JL et al. cAMP response element-binding (CREB)

this is simply a bystander tissue [18]. A synovial joint contains many other tissues besides cartilage including the subchondral bone, synovium, periarticular muscles, ligaments and meniscus (if present), all of which, to a greater or lesser extent, are implicated in OA induction or progression. Perhaps before we embark on these complex epigenetic analyses we need to be sure which tissue should be the focus of our research.

\section{Disclaimer}

The views expressed are those of the authors and not necessarily those of the NHS, the NIHR or the Department of Health.

\section{Financial \& competing interests disclosure}

This work in the authors laboratory is supported by funding from by Arthritis Research UK grants numbers 18746 and 19424, the JGWP Foundation and the Newcastle University Hospitals Special Trustees, UK. The research was supported by the National Institute for Health Research (NIHR) Newcastle Biomedical Research Centre based at Newcastle-upon-Tyne Hospitals NHS Foundation Trust and Newcastle University. The authors have no other relevant affiliations or financial involvement with any organization or entity with a financial interest in or financial conflict with the subject matter or materials discussed in the manuscript apart from those disclosed.

No writing assistance was utilized in the production of this manuscript.

recruitment following a specific $\mathrm{CpG}$ demethylation leads to the elevated expression of the matrix metalloproteinase 13 in human articular chondrocytes and osteoarthritis. FASEB J. 26(7), 3000-3011 (2012).

8 Zhang Y, Jordan JM. Epidemiology of osteoarthritis. Clin. Geriatr. Med. 26(3), 355-369 (2010).

9 arcOGEN Consortium, arcOGEN Collaborators. Identification of new susceptibility loci for osteoarthritis (arcOGEN): a genome-wide association study. Lancet 380(9844), 815-823 (2012).

10 Feng S, Jacobsen SE, Reik W. Epigenetic reprogramming in plant and animal development. Science 330(6004), 622-627 (2010).

11 Hackett JA, Zylicz JJ, Surani MA. Parallel mechanisms of epigenetic reprogramming in the germline. Trends Genet. 28(4), 164-174 (2012).

12 Bell JT, Tsai PC, Yang TP et al. Epigenomewide scans identify differentially methylated regions for age and age-related phenotypes in a healthy ageing population. PLoS Genet. 8(4), e1002629 (2012).
13 Meaburn EL, Schalkwyk LC, Mill J. Allele-specific methylation in the human genome: implications for genetic studies of complex disease. Epigenetics 5(7), 578-582 (2010).

14 Kong A, Steinthorsdottir V, Masson G et al. Parental origin of sequence variants associated with complex diseases. Nature 462(7275), 868-874 (2009).

15 Bell CG, Beck S. The epigenomic interface between genome and environment in common complex diseases. Brief Funct. Genomics 9(5-6), 477-485 (2010).

16 Reynard LN, Bui C, Canty-Laird EG, Young DA, Loughlin J. Expression of the osteoarthritis-associated gene GDF5 is modulated epigenetically by DNA methylation. Hum. Mol. Genet. 20(17), 3450-3460 (2011).

17 Bernstein BE, Birney E, Dunham I, Green ED, Gunter C, Snyder M. An integrated encyclopedia of DNA elements in the human genome. Nature 489(7414), 57-74 (2012).

18 Brandt KD, Radin EL, Dieppe PA, van de Putte L. Yet more evidence that osteoarthritis is not a cartilage disease. Ann. Rheum. Dis 65(10), 1261-1264 (2006). 